University of Nebraska - Lincoln

DigitalCommons@University of Nebraska - Lincoln

January 1986

\title{
Biological Effects of Heavy lons From the Standpoint of Target Theory
}

Robert Katz

University of Nebraska-Lincoln, rkatz2@unl.edu

Follow this and additional works at: https://digitalcommons.unl.edu/physicskatz

Part of the Physics Commons

Katz, Robert, "Biological Effects of Heavy lons From the Standpoint of Target Theory" (1986). Robert Katz Publications. 74.

https://digitalcommons.unl.edu/physicskatz/74

This Article is brought to you for free and open access by the Research Papers in Physics and Astronomy at DigitalCommons@University of Nebraska - Lincoln. It has been accepted for inclusion in Robert Katz Publications by an authorized administrator of DigitalCommons@University of Nebraska - Lincoln. 


\title{
BIOLOGICAL EFFECTS OF HEAVY IONS FROM THE STANDPOINT OF TARGET THEORY
}

\author{
Robert Katz \\ University of Nebraska-Lincoln, Lincoln, NE 68588-111, U.S.A.
}

\begin{abstract}
The biological effect of heavy ions is best described through the action cross section, as a function of the endrpoint of interest and the charge and speed of the ion. In track theory this is called the "ion-kill" cross section, for it is the effect produced by a single heavy ion and its delta rays. As with nuclear emulsions the biological track structure passes from the grain count regime to the track width regime to the thindown region with an increase in LET. With biological cells, as with any detector capable of storing sub-lethal damage, with low LET irradiation the action cross section (in the ion-kill mode) is increasingly obscured by the effect of "gammar kill", by the influence of overlapping delta rays from neighboring heavy ions. Thus at low LET response is dominated by the gamma-kill mode, so that the RBE approaches 1. The theory requires 4 radiosensitivity parameters for biological detectors, extracted from survival curves at several high LET bombardments passing through the grain count regime, and at high doses. once these are known the systematic response of biological detectors to all high LET bombardments can be unfolded separating ion kill from gamma kill, predicting the response to a mixed radiation environment, and predicting low dose response even at the level of a single heavy ion. Cell killing parameters are now available for a variety of cell lines. Newly added is a set of parameters for cell transformation.
\end{abstract}

\section{INTRODUCTION}

It is somewhat surprising that a simple model of the structure of a particle track based upon target theory can encompass the response of a large number of different detectors to energetic heavy ions, though these detectors have little apparent similarity. It is even more surprising that, given that 3 parameters suffice for describing the relative response of many physical detectors, only a single additional parameter can yield a description of the response to heavy ions of biological cells in vitro, though these are surely enormously more complex than an alanine molecule, or a virus, or a scintillation counter, or a TLD crystal, or a dye film, or a nuclear emulsion, or a Fricke dosimeter. The model does not incorporate time dependent or dose rate effects. This would require at least one additional parameter.

The model extends target theory to energetic heavy ions.

Target theory assumes that the response of a detector to gamma rays takes the statistical form of the cumulative poisson distribution, as a result of fluctuations in energy deposition. No further introduction of fluctuation is required. Taking as a detector parameter the characteristic dose $E_{0}$ at which there is an average of 1 hit per target, the number of poisson trials, $A$, at dose $D$ is then $A=D / E_{0}$, and the probability of success $P$ in a situation where $C$ or more hits are required is $P(C, A)$, according to the Poisson formula. For a 1 hit detector this leads to the usual exponential form of a survival curve. The parameters required to describe such a detector are 3 in number: 1) the radius of the target, $a_{0}, 2$ ) the characteristic dose, $E_{0}$, and 3) the minimal number of hits, C, required to activate the detector. Most detectors are 1ror-more hit detectors; that is, $C=1$. If a single 
electron passing through a target can activate it, we say that the detector is 1-hit. Such detectors tend to discriminate against high LET radiations. The RBE of such detectors for high LET radiations is never greater than 1 , and can be down by 2 orders of magnitude. Many hit detectors require more than 1 electron through a sensitive target for activation. They thus accumulate sub-lethal damage or sub-latent image. They tend to discriminate in favor of high LET radiations. The RBE of such detectors can be substantially greater than 1. Blological cells are a special variant. We think of them as if they were made up of a collection of 1-hit targets within the nucleus. The cell is then thought to be inactivated if some number of these are inactivated by the radiation field. But more of this later.

What is a hit? At most we can say that a hit is an interaction of a secondary electron with a detector which leads to the observed action. We must expect electron interactions to be energy dependent. Thus our characteristic parameter $E_{0}$ may depend somewhat on the electron energy spectrum thus giving rise to variations in RBE for low LET radiations of different quality. But the dependence is typically a weak one and we tend to assign it a low priority.

THE ACTION CROSS-SECTION

To describe the response of the detector to heavy ions we need to calculate the action cross-section.

Let us remind ourselves of the meaning of the cross-section. Imagine that a single ion is passing down a channel $1 \mathrm{~cm}^{2}$ in area, and that it may interact with a single target somewhere in that channel. The objective probability that such an interaction takes place, given as the fraction of successes in a large number of identical repeated trials, is written as the ratio of the cross-section to the area of the channel. We note that the cross-section is an average quantity, an expectation value. The cross-section represents a probability. It may be very much smaller or larger than the geometric cross-section of the target.

Let us connect the concept of cross-section with the appearance of a particle track in emulsion. Suppose that every grain intersected by an ion is made developable and no grains off the ion's path are so affected. In this circumstance the track looks like a tight strand of single pearls. Here the cross-section is $\sigma_{0}$. A particle of lesser charge or greater speed makes a track that looks like randomly gapped pearls. We call this the grain-count regime. The cross section $\sigma$ is smaller than $\sigma_{0}$. If $N$ is the number of undeveloped grains $/ \mathrm{cm}^{3}$, then the linear density of developed grains is $\sigma \mathrm{N}$. These grains are not required to be along the ion's path, but are most likely to be there because of the nature of the energy deposition. If we now consider an ion of higher charge which generates more delta rays that penetrate grains off the ion's path the track may look like a hairy rope. We call this the track-width regime. The cross-section may be approximated as the average cross-sectional area of the rope. It is now greater than $\sigma_{0}$, perhaps orders of magnitude greater than the geometric cross sectional area of the target.

In the grain count regime the cross section, the linear density of activated targets along a track, always increases with an increase in LET, as the particle slows down.

In the track width regime kinematic limitations on delta ray energies play tricks with us. As the ion slows down the track first increases in width to a maximum value not at all related to the maximum in energy deposition, and then thins down like a sharpened pencil as the track appearance is dominated by the kinematic limit in delta ray energies. There are many delta rays. There is much energy loss. But the cross section diminishes with an increase in LET as the rope thins down. Whenever it is observed that the crosssection diminishes with an increase in LET we may be certain that we are in the track width regime, and that energetic delta rays play a dominant part in the production of the action cross-section. Thin down effects have been observed in several detectors, including nuclear emulsion, scintillation counters, thermoluminescent dosimeters, and possibly (but not certainly) CR-39, the plastic widely used for etchable track detection. They have been observed at GSI, Darmstadt, with mammalian and yeast cells and bacterial spores. There can no longer be doubt of the central importance of delta rays in the response of biological cells to high LET radiations. At the same 
R. Katz, Biological Effects of Heavy lons, in Adv. Space. Res. 6 (1986)

time we see that LET can be a confusing parameter for it does not reflect the energy spectrum of delta rays.

To calculate the action cross-section we need to know the average radial distribution in "local dose" about an ion's path. The determination of the radial dose distribution, from all ions at all speeds, in all media of interest has required a sustained effort over the years, and is far from complete. But it is central to the theory. Our best present knowledge of the radial dose distribution in water comes a combination of monte carlo calculations for liquid water and experimental data for a variety of gases, for proton bombardment. We do not have good information about the dose at the maximum radial delta ray penetration. Nor do we have good information for energetic heavy ions, especially when partiy clothed with electrons, nor about other media than water. For heavy ions we use a stopping power based effective charge formula to scale the proton calculation though we are aware that in general effective charge is basically an invalid concept. For other media we scale by density. A great deal more knowledge about the interaction of heavy ions with matter is required than is presently available.

Again, knowledge of the microdosimetric dose fluctuations are of no particular value to this model for they are an implicit part of the dose-response curve measured with gammarrays. That dose-response curve, interpreted probabilistically, is now applied to the radial dose distribution to yield the radial distribution in the probability that targets will be "activated". If at radial distance $t$ the probability for activation about the path of an "average particle" is $P(t)$, we find the cross section as the radial integral of this probability. We base the radial probability upon the average energy per unit volume in a target of radius $a_{0}$ whose center is at radial distance $t$ from the ion's path, taking yet another average because the dose falls off rapidly with distance, nearly quadratically. The gamma-ray dose-response curve is taken as a calibration of the response of the targets of which the detector is made, of what the detector experiences as a hit. Ideally one should like to be able to match the electron energy spectrum in different shells about the ion's path with an identical calibrating spectrum, but this is not possible. It is therefore taken as a adequate approximation that in most cases the electron slowing down spectrum from gamma rays dose not significantly differ from that from delta rays in the different shells. In principle we imagine that we collect the radiation fields from identical shells about the paths of different isolated ions into a volume sufficiently large that the concept of macroscopic dose is meaningful.

Note then that this procedure enables us to make two kinds of comparisons with experimental data. Having a radial distribution in effect we are able to produce computer simulations of the tracks of individual particles in emulsion. Having the cross section enables us to make comparison with measurements which arise from beams of particles in homogeneous track segment irradiation.

\section{BIOLOGICAL CELLS}

Biological cells present a perplexing structure. We cannot be certain of the number, the location, the radiosensitivity of any of the targets whose activation leads to an observed end-point. For the present work we have taken the nucleus of the cell to be the volume within which the targets are contained, rather like beans in a bean bag. The beans are taken to be 1-hit detectors, of which some number, $m$, must be activated to yield the endpoint. We calculate as if the required aggregate of activated beans were a single m-target, 1-hit per target, detector of radius $a_{0}$, for which each bean had a characteristic dose $E_{0}$. For these targets we calculate a cross section $S$. But since these targets may be intersected by heavy ions "anywhere" within the nuclear volume we take it that the observed action crosssection $\sigma$ is larger than the target cross-section approximately in the ratio of the cross sectional area of the bean bag to the cross sectional area of the bean. We must keep in mind however that the action cross-section represents a probability rather than a geometric area. If for a particular end point to be activated there is a time window in the life of the cell, then, for a heterogeneous population of cells the cross-section may be appropriately reduced. The properties of the bean then determine the location of the grain count regime, the track width regime, and the onset of thin-down. While we identify the bean bag with the nuclear volume we note that this identification is a loose one. We do not know how the beans cluster within the nucleus. The basis for the identification of a biological 
track width regime is not the relationship of the action cross-section to the size of the nucleus, but rather the observation of thin-down. The number of our parameters must be expanded by 1 over the physical detector, for we need to include the size of the bean bag. Thus for biological cells we require 4 fitted parameters. As incorporated in the cell survival equations used in our formulation, reviewed in the next section, these are $E_{0}$, $\mathrm{m}, \sigma_{0}$, and $k$ which is approximately given as $\mathrm{E}_{0} \mathrm{a}_{0}{ }^{2} / 2 \times 10^{-}, \mathrm{cm} / \mathrm{erg}$.

It is astonishing that so few parameters enable us to describe the response of biological cells to energetic heavy ions. These parameters must be evaluated from a set of survival curves spanning the grain count regime by fitting the model to experimental data. Once they are known the action cross sections for a particular cell line and end point may be calculated at all bombardments, from the lowest to the highest LET, within the uncertainty set by our lack of knowledge of the radial dose distribution.

THE MODEL

Detalled descriptions of the cellular track model have been given elsewhere(1). Here we present only the main concepts of the model and list the equations used in our calculations. Following our earlier studies of the appearance of particle tracks in nuclear emulsion(2), the model distinguishes between the "grain-count" regime where inactivat fons occur randomly along the particle's path, and the "track width" regime where the inactivations are distributed like a "hairy rope". The transition from the graincount to track width regime takes place in the neighbourhood of $Z^{*} 2 / \mathrm{K}^{2}$ of about 4 ; at lower values we are in the grain-count regime, at higher values in the track width regime.

To accomodate for the capacity of cells to accumulate sub-lethal damage, two modes of inactivation are identified, namely "ion-kill" (or "intra-track") and "gammarkill" (or "inter-track"). In these two inactivation modes it is the statistical character of the inactivation which is changing rather than the fundamental physical interaction. Effects are referred to dose rather than to the number of electrons passing through the nucleus.

Cells inactivated by the passage of a single heavy ion are said to be inactivated in the ion-kill mode with inactivation cross-section $\sigma$ whose value is less than $\sigma_{0}$ in the grain-count regime and greater than $\sigma_{0}$ in the track width regime. The fraction of "track segments" inactivating cells in the ion-kill mode in the grain-count regime is taken to be $\sigma / \sigma$ o which is equal to $P$, the probability for inactivation in the ion-kill mode, $P$ also approximates the fraction of the dose deposited in the ion-kill mode. The RBE goes through a maximum when $P-0.5$. Cells not inactivated in the ionkill mode can be sublethally damaged by the $\delta-r a y s$ from the passing particle and then inactivated, in the gamma-kill mode, by cumulative addition of sublethal damage due to orrays from other passing ions. Survival in the gammarkill mode is taken to follow the m-target statistics of inactivation by secondary electrons from Xrray or gammarray photons.

In the grain-count regime the surviving fraction of a cellular population whose radiosensitivity parameters are $m, E_{0}, \sigma_{0}$ and $k$, after track-segment irradiation with an ion dose ${ }_{*}$ of a fluence of $F$ particles of atomic number $Z$, effective charge value $Z^{*}$, relative speed $\beta$ and stopping power $L$ (LET $)_{\infty}$, is found from the expressions:

$$
\frac{\mathrm{N}}{\mathrm{N}_{0}}=\Pi_{i} \times \Pi_{\gamma}
$$

where the ion-kill mode survival probability is:

$$
\Pi_{i}=\exp (-\sigma F)
$$

the gamma-kill mode survival probability is:

and the gammarkill dose fraction is:

$$
\Pi_{\gamma}=1-\left[1-\exp \left[\frac{-D_{\gamma}}{E_{0}}\right]\right)^{m}
$$

$$
D_{\gamma}=(1-P) D
$$


R. Katz, Biological Effects of Heavy lons, in Adv. Space. Res. 6 (1986)

where

$$
\frac{\sigma}{\sigma_{0}}=P=\left[1-\exp \left[\frac{-z^{* 2}}{k \beta^{2}}\right]\right]^{m}
$$

In the track-width regime, where $P>0.98$, we take:

$$
\Pi_{\gamma}=1
$$

and find $\sigma$ from the "track width" which increases fineąrly with $Z^{*} / \beta$ while the inactivation cross-section increases with $Z^{*} / \beta^{2}$ up to a limit set by the maximum radial range of $\delta$-rays. This is the "thindown" region( 3$)$.

To calculate RBE at a given "kill" (transformation) or survival level we use the definition:

$$
R B E=\frac{D_{x}}{D}
$$

where

$$
D_{X}=-E_{0}\left[\ln \left(1-\left(1-N / N_{0}\right)^{1 / m}\right)\right]
$$

is the X-ray dose after which this level obtains, and $D$ is the corresponding ion dose.

All our calculations pertain to water so the ion dose is always

$$
D=F L
$$

When "cross-sections" and RBE's are calculated from the final slope of the survival curves, we refer to the crossesection and the RBE as "extrapolated" and in the grain count regime we write

$$
\sigma_{\text {ext }}=\sigma_{0} P+(1-P) L / E_{0}
$$

and

$$
R B E_{\text {ext }}=\left(\sigma_{0} E_{0} / L\right) P+(1-P)
$$

To calculate the effective charge value of an ion of atomic number $z$ moving with a relative velocity $\beta$ we use the expression:

$$
Z^{*}=Z\left[1-\exp \left(-125 \beta Z^{-2 / 3}\right)\right]
$$

We calculate the stopping power and range in water of an ion of atomic number $Z$ with the aid of Janni's proton stopping power and range tables (4) and the expressions:

$$
L(Z, \beta)=L(p, \beta)\left[Z^{*} / z_{p}^{*}\right]^{2}
$$

where $Z^{*}$ and $Z^{*}$ are the effective charges of the ion and proton, respectively (from $\mathrm{kq} .11$ ) and $\mathrm{L}(\mathrm{p}, \beta)$ is the stopping power, in water, of a proton at the same speed $B$, and

$$
R(Z, \beta)=\left(A / Z^{2}\right)\left[R_{p}(B)+C(B)\right]
$$

where $A$ is the ion mass number (in $a m u), R_{p}(\beta)$ is the range, in water, of a

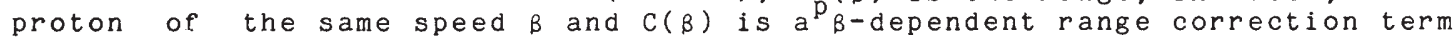
after Barkas and Berger(5).

Simple programs for calculating track-segment "kill" and survival using eqs. $(1-10)$, and for calculating stopping power and range for ions in water (eqs.(13-14)) were developed, also for the HP-41C hand-held calculator (M.P.R. Waligorski, unpublished 1985).

Our treatment of transformations uses the same set of equations, and takes cell killing and transformation to be independent processes that take place along the same particle track. We require separate sets of parameters for cell killing, and for transformation. These have been fitted to the data of 
Yang $(6)$.

RESULTS

These models have unifled our understanding of the variation in response with LET of a large number of physical detectors and biological systems from a single perspective, essentially that detectors can be imagined to be an accumulation of targets that may or may not accumulate damage, and that one can map the response to gamma rays into the radial dose distribution about an ion's path. The models seem to relate not only to detector response, and that of biological systems, but also to such diverse questions as soft errors in computer systems from cosmic rays, heavy ion writing on photoresists for computer chip production, and the desorption of large organic molecules from a substrate for purposes of mass spectrometry. They are now described in several textbooks. They have stimulated a considerable amount of investigation into the structure of particle tracks and related atomic physics.

The models are phenomenological and parametric. Though it must not be imagined that the construction of a general model of radiation response with 3 or 4 parameters is a trivial undertaking. I have heard many cautionary lectures about fitting data with 4 parameters but note that no competitive model of comparable scope has emerged though the present model has been exposed for 20 years.

Qualitatively our models have shown us many things:

1) There can be no single parameter reduction of a radiation field which predicts radiation response. Such parameters as the average LET of a mixed radiation field are particularly misleading. In fact when RBE is plotted against average LET in a range modulated heavy ion beam, the heaviest particles yield the highest RBE. But when these plots are made for track segment irradiations the lightest particles display the highest RBE.

2) The properties of a detector and the properties of the radiation field are not separable variables; that is, one cannot describe detector response as a product of two factors, one of which contains only parameters of the radiation and the other containing only parameters of the detector. Thus it is nonsense to speak of the RBE of neutrons, or the quality factor of alpha particles without regard to the endrpoint or the dose level.

3) Radiation effects are statistical in character. There are no thresholds of radiation response. All radiation effects are stochastic in character. It is meaningless to speak of energy deposition thresholds of order several hundred $\mathrm{eV}$. for the initiation of biological action in a cell.

4) Delta rays are the principle agency for the interaction of an energetic heavy ion with the targets of a detector. Since LET ignores the energy spectrum of delta rays we find that response (RBE, action cross-section, extrapolated cross-section) are multiple valued functions of LET. Different ions moving at different speeds, but having the same LET, produce widely different response in the same detector. Clear unequivocal evidence for the importance of delta rays lies in the observation of "thindown", the decline in cross-section with an increase in LET observed in physical and biological detectors for heavy ions approaching the end of their range.

Qualitative predictions of a biological track width regime, and of biological thin-down were made some 15 years ago from track theory. Some recent quantitative findings (3) are shown in Fig. 1. Cellular parameters derived that long ago from survival data obtained on the Berkeley HILAC with chinese hamster cells were analyzed to produce the target parameters of the "bean within the bean bag". This was combined with a calculated expression of the radial distribution of dose about the path of a heavy ion to yield a calculation of the action cross section $S$ of the bean for different ions and ion speeds. This in turn was multiplied by a factor $9 x$ (approximately the ratio of the area of the bean bag to the area of the bean) for comparison with experimental measurements made with ions up to uranium at the GSI UNILAC, Darmstadt. The initial calculation did not agree well with experiment, the calculated "hooks" for the heavy element bombardment lying away from the experimental data. This was attributed to an error in the radial dose formulation, where the maximum radial penetration of delta rays was assumed to be as if the most energetic delta rays were ejected normally. By adjustment 
R. Katz, Biological Effects of Heavy lons, in Adv. Space. Res. 6 (1986)

it was found that if the maximum radial penetration as initially calculated was reduced by factor 5 the results shown in Fig 1 were obtained. Experimental data giving the maximum radial penetration is not available. For one case, that of energetic He ions a Monte Carlo calculation had been made by Paretzke, for comparison with some experimental data by Varma and collaborators. As shown in Fig. 2 this served to justify (somewhat) the factor 5 reduction in maximum delta ray range.

In Fig. 1 the solid lines are the calculated action (ion-kill) cross sections. The dotted lines at left are the extrapolated cross-sections calculated from Eq. (10). The large data points are from the earlier HILAC bombardments, while the smaller data points are from the later UNILAC bombardments.

5) The fluctuation in energy deposition is an integral part of the doseresponse curves of target theory. To the accuracy of present data we have no need for additional consideration of the fluctuation in energy deposition. Our calculations are all based on averages. Their result is the prediction of an average response.

6) The concept of a "Quality Factor" is based on false premises. It does not properly describe the response of any biological system, for it ignores differences in relative response at different dose levels, it assumes the separability of a parameter supposed to describe the radiation field, the LET, from the biological parameters, it ignores thindown and multiple valuedness of response to different ions at the same LET. Its errors cannot be corrected by altering its numerical value. A totally new conceptual structure is required.

7) Relative to the question of quality factor, the goal is to produce a calculated dose of gamma-rays which produce the same response as a given dose of specified high LET radiations. Track theory can do this for cell killing, and now possibly for one variety of transformation. It has been shown that RBE-dose relationships obtained for several in-vivo end-points with neutrons are well matched by calculated values for several cell killing parameter sets. The mathematics for obtaining the equivalent gammarray dose can be programmed on a hand held calculator. It seems reasonable to propose that track theory be made the basis of a revised "quality factor".

\section{ACKNOW LEDG EM ENT}

This work is sponsored by the United States Department of Energy.

\section{$\underline{\text { References }}$}

1. R. Katz, "Track Structure Theory in Radiobiology and in Radiation Detection," Nucl. Track Detection 2,1-28 (1978).

2. R. Katz and E. J. Kobetich, "Particle Tracks in Emulsion," Phys. Rev. $186,344-351$ (1969).

3. R. Katz, D. E. Dunn, and G. L. Sinclair, "Thindown in Radiobiology," Radiat. Prot. Dosimetry 13,281-284 (1985).

4. J. F. Janni, "Proton Range-Energy Tables, 1 KeV - 10 GeV," At. Data and Nucl. Data. Tables 27,147-529 (1969).

5. W. H. Barkas and M. J. Berger, "Tables of Energy Losses and Ranges of Heavy Charged Particles," pp. 103-172 in Studies in Penetration of Charged Particles in Matter, N.A.S., N.R.C., washington D. C. (1964).

6. T. C. Yang, L. M. Craise, and C. A. Tobias, "Neoplastic Cell Transformation by Heavy Charged Particles," Radiat. Res. Suppl. 104,S-177-S187 (1985). 
Fig. 1. From cellular radiosensitivity parameters fitted to the survival data for Chinese Hamster cells in 1971, inactivation cross- sections were calculated for the hypothetical sub-nuclear target a appropriate to these cells. These cross sections, $S$, are converted to cellular action crosssection by multiplication by factor 9 , being approximately the ratio of $\sigma_{0}$ to $S_{0}$, the plateau value of the measured cellular cross-section to the calculated cross-section for the hypothetical target. To obtain better agreement between the calculated and observed cross-sections the maximum radial delta ray penetration was reduced by factor 5 from the value obtained from assuming normal ejection.

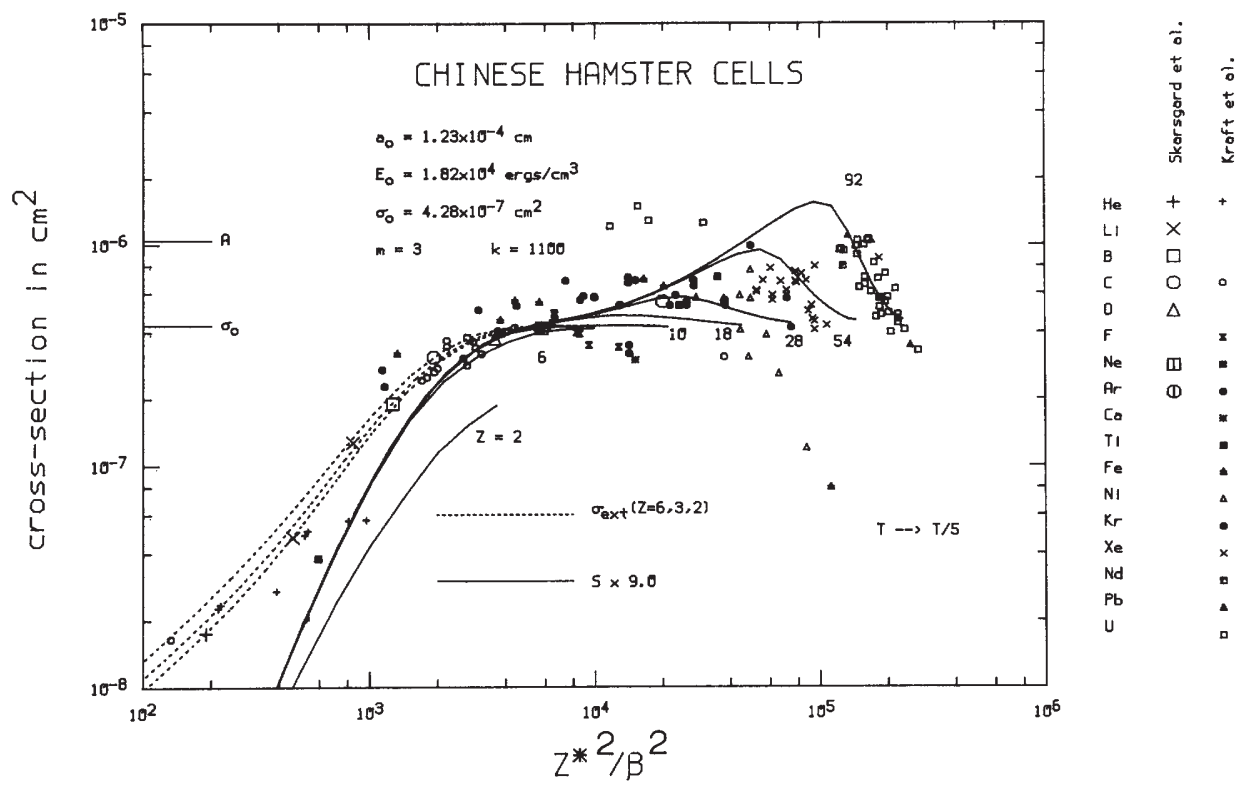

Fig. 2. A comparison of the radial distribution of dose for fast lle ions measured by Varma and collaborators shown as squares, a Monte carlo calculation by paretzke identified by $P$, a distribution calculated from the assumption of normal ejection of the most energetic delta rays, $T$, and one calculated from the assumption that the range of the most energetic delta rays is reduced by factor 5, T/5. The latter assumption lies closer to the Monte Carlo calculation of Paretzke than the original one, providing some justification for its use in Fig. 1 .
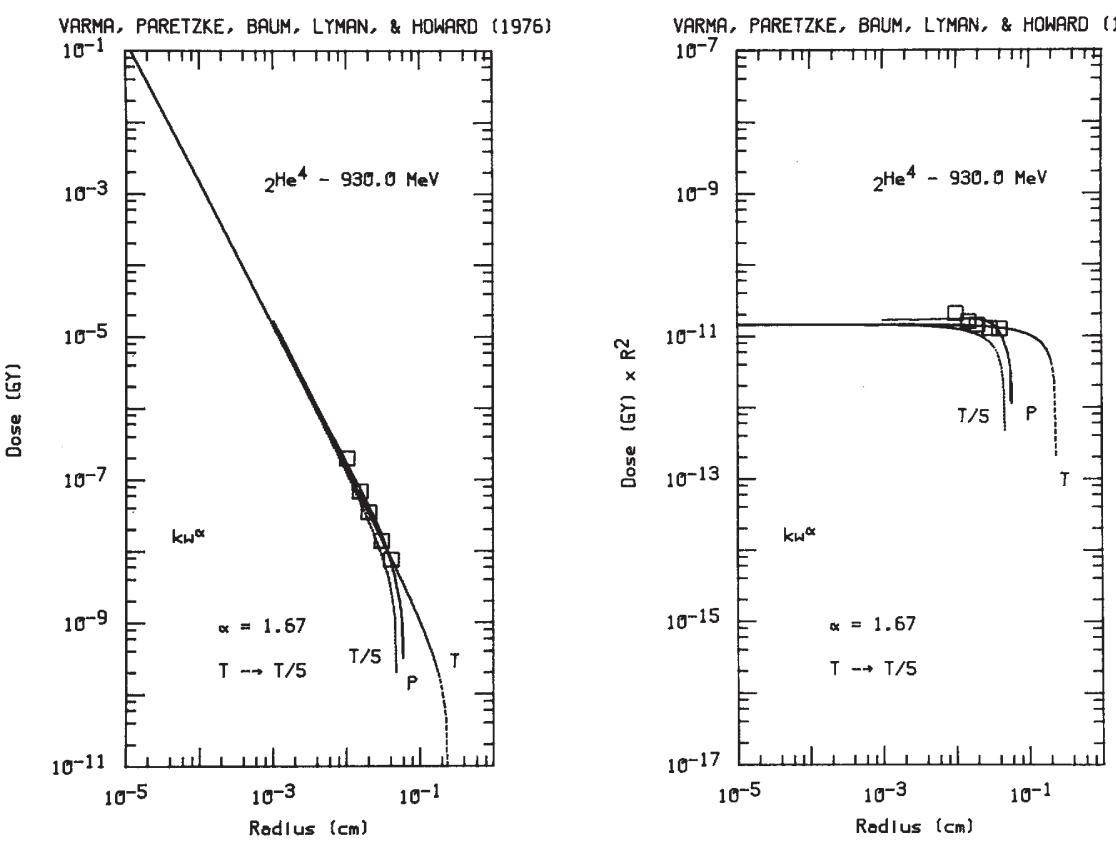